\title{
LIFTING HOMEOMORPHISMS OF A COMPLEX SURFACE TO ITS NORMALIZATION
}

\author{
WILLIAM A. ADKINS \\ (Communicated by Clifford J. Earle, Jr.)
}

\begin{abstract}
It is proved that any homeomorphism of a complex analytic surface lifts to a homeomorphism of the normalization.
\end{abstract}

The purpose of the present note is to provide a proof of the following result which was mentioned in the paper [3] of Steenbrink and Stevens without proof.

Theorem. Any homeomorphism of a complex analytic surface lifts to a homeomorphism of the normalization.

Before giving the proof of this theorem we recall the basic notations and results which are needed. By a complex analytic surface we mean a reduced, second countable, complex space of pure dimension 2 . If $\left(X, \mathscr{O}_{X}\right)$ is a complex space with structure sheaf $\mathscr{O}_{X}$ and $a \in X$, then $(X, a)$ will denote the germ of $X$ at $a$. A theorem of Mumford [2] states that if the germ $(X, a)$ is homeomorphic to the germ $\left(\mathbf{C}^{2}, 0\right)$, then $a$ is a nonsingular point of $X$.

If $X$ is a complex surface, then $S(X)$ will denote the singular subspace of $X$ with the reduced structure. We say that a point $a \in S(X)$ is a topological multicross if the germ $(X, a)$ can be written as a union of surface germs $\left(X_{i}, a\right)$

$$
(X, a)=\left(X_{1}, a\right) \cup \cdots \cup\left(X_{r}, a\right)
$$

where

(1) $\left(X_{i}, a\right)$ is analytically isomorphic to $\left(\mathbf{C}^{2}, 0\right)$ for $1 \leq i \leq r$.

(2) $\left(X_{i}, a\right) \cap\left(X_{j}, a\right)=(S(X), a)$ for all $i \neq j$.

(3) $(S(X), a)$ is homeomorphic to $(\mathbf{C}, 0)$

A point $a \in S(X)$ is said to be an analytic multicross, or just a multicross, if the germ $(X, a)$ satisfies conditions (1), (2), and the following condition $\left(3^{\prime}\right)$, instead of $(3)$.

$\left(3^{\prime}\right)(S(X), a)$ is analytically isomorphic to the germ $(\mathbf{C}, 0)$.

The multicross singularities of complex surfaces were introduced in [1] in order to provide a structure theorem for weakly normal surface singularities.

Received by the editors May 26, 1989 and, in revised form, October 2, 1989.

1980 Mathematics Subject Classification (1985 Revision). Primary 32C20, 32C40. 
Recall that a complex space is weakly normal if the sheaf $\mathscr{O}_{X}^{c}$ of germs of $c$ holomorphic functions on $X$ is equal to the sheaf $\mathscr{O}_{X}$ of germs of holomorphic functions on $X$.

We now introduce notation for certain subsets of $X: X_{\text {reg }}$ denotes the set of all regular (smooth) points of $X, X_{t m}$ denotes the set of all topological multicross points of $X$, and $X_{a m}$ denotes the set of all analytic multicross points of $X$. Then we let $X_{s}=X \backslash\left(X_{\text {reg }} \cup X_{t m}\right)$ and $X_{u}=X \backslash\left(X_{\text {reg }} \cup X_{a m}\right)$. Thus $X$ can be written as a disjoint union in two different ways: $X=X_{\text {reg }} \cup X_{t m} \cup X_{s}=$ $X_{\text {reg }} \cup X_{a m} \cup X_{u}$. Since $X_{a m} \subseteq X_{t m}$, it follows that $X_{s} \subseteq X_{u}$. If $X$ is a weakly normal complex surface, then the Oka theorem for weakly normal singularities (Theorem 2.4 of [1]) shows that $X_{u}$ (and hence $X_{s} \subseteq X_{u}$ ) is a discrete subset of $X$.

Lemma. Let $X$ be a complex surface and let $h: X \rightarrow X$ be a homeomorphism. Then $h\left(X_{\text {reg }}\right)=X_{\text {reg }}, h\left(X_{t m}\right)=X_{t m}$, and $h\left(X_{s}\right)=X_{s}$.

Proof. If $a$ is a regular point of $X$, then $h(a)$ is a regular point of $X$ by Mumford's theorem. Thus $h\left(X_{\mathrm{reg}}\right)=X_{\mathrm{reg}}$. Suppose $a \in X_{t m}$. Then $(X, a)=$ $\left(X_{1}, a\right) \cup \cdots \cup\left(X_{r(a)}, a\right)$ where $\left(X_{i}, a\right)$ is analytically isomorphic to $\left(\mathbf{C}^{2}, 0\right)$ and $\left(X_{i}, a\right) \cap\left(X_{j}, a\right)=(S(X), a)$ for all $i \neq j$ and with $(S(X), a)$ homeomorphic to $(\mathbf{C}, 0)$. Now $h:(X, a) \rightarrow(X, h(a))$ is a homeomorphism of germs and $h(S(X))=S(X)$ so that $h:(X \backslash S, a) \rightarrow(X \backslash S, h(a))$ is also a homeomorphism of germs, where $S(X)=S$. Therefore, $(X \backslash S, h(a))$ has the same number of connected components as $(X \backslash S, a)$, so that $(X, h(a))$ can be written as a union $\left(X_{1}^{\prime}, h(a)\right) \cup \cdots \cup\left(X_{r(a)}^{\prime}, h(a)\right)$ and $h:(X, a) \rightarrow(X, h(a))$ decomposes (after reindexing, if necessary) into a union of homeomorphisms $h_{i}:\left(X_{i}, a\right) \rightarrow\left(X_{i}^{\prime}, h(a)\right)$ with the property that $\left.h_{i}\right|_{X_{i} \cap X_{j}=S(X)}=\left.h_{j}\right|_{X_{i} \cap X_{j}=S(X)}$. By Mumford's theorem, $\left(X_{i}^{\prime}, h(a)\right)$ is nonsingular for all $i$, and $(S(X), h(a))$ is homeomorphic to $(S(X), a)$, which is homeomorphic to $(\mathbf{C}, 0)$. Therefore, $h(a) \in X_{t m}$. Finally, $h\left(X_{s}\right)=X_{s}$ since $X$ is a disjoint union of $X_{\text {reg }}, X_{t m}$, and $X_{s}$.

We now proceed to the proof of the theorem. Thus let $X$ be a complex analytic surface, let $\eta: \widetilde{X} \rightarrow X$ be the normalization of $X$, and let $\widetilde{X}_{\text {reg }}=$ $\eta^{-1}\left(X_{\text {reg }}\right), \tilde{X}_{t m}=\eta^{-1}\left(X_{t m}\right)$, and $\tilde{X}_{s}=\eta^{-1}\left(X_{s}\right)$. Assume that $h: X \rightarrow X$ is a homeomorphism. Then we claim that there is a homeomorphism $\tilde{h}: \tilde{X} \rightarrow \tilde{X}$ such that the diagram

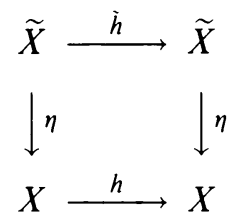

is commutative. The proof proceeds by constructing the homeomorphism $\tilde{h}: \widetilde{X} \rightarrow \widetilde{X}$ successively on $\widetilde{X}_{\text {reg }}, \widetilde{X}_{t m}$, and $\widetilde{X}_{s}$. Without loss of generality, 
we may assume that $X$ is weakly normal (since the weak normalization $X^{w}$ of $X$ is homeomorphic to $X$ [1]). As observed above, $X_{s}$ is a discrete subset of $X$ and since $\eta: \widetilde{X} \rightarrow X$ is a finite map, $\widetilde{X}_{s}$ is a discrete subset of $\widetilde{X}$. Also note that $\widetilde{X}_{s}=\eta^{-1}\left(h\left(X_{s}\right)\right)$ since, according to the lemma, $h\left(X_{s}\right)=X_{s}$.

Define $\tilde{h}^{\prime}: \widetilde{X}_{\text {reg }} \rightarrow \widetilde{X}_{\text {reg }}$ by $\tilde{h}^{\prime}=\bar{\eta}^{-1} \circ h \circ \bar{\eta}$ where $\bar{\eta}=\left.\eta\right|_{\widetilde{r}_{\text {reg }}}: \widetilde{X}_{\text {reg }} \rightarrow \widetilde{X}_{\text {reg }}$ is biholomorphic. It remains to extend $\tilde{h}^{\prime}$ homeomorphically across $\widetilde{X}_{t m} \cup$ $\tilde{X}_{s}$. Suppose that $a \in X_{t m}$. According to the lemma $(X, a)=\left(X_{1}, a\right) \cup$ $\cdots \cup\left(X_{r(a)}, a\right)$ where each $\left(X_{i}, a\right)$ is analytically isomorphic to $\left(\mathbf{C}^{2}, 0\right)$ and $\left(X_{i}, a\right) \cap\left(X_{j}, a\right)$ is homeomorphic to $(\mathbf{C}, 0)$ whenever $i \neq j$, with a similar expression for $h(X, a)=\left(X^{\prime}, h(a)\right)=\left(X_{1}^{\prime}, h(a)\right) \cup \cdots \cup\left(X_{r(a)}^{\prime}, h(a)\right)$. Let $h_{i}=\left.h\right|_{\left(X_{i}, a\right)}$. Then $h_{i}$ is a homeomorphism from $\left(X_{i}, a\right)$ to $\left(X_{i}^{\prime}, h(a)\right)$. Since $\eta^{-1}(a)=\left\{b_{1}, \ldots, b_{r(a)}\right\}$ with $\bar{\eta}_{i}=\left.\eta\right|_{\left(\widetilde{X}, b_{i}\right)}:\left(\tilde{X}, b_{i}\right) \rightarrow\left(X_{i}, a\right)$ biholomorphic and $\eta^{-1}(h(a))=\left\{c_{1}, \ldots, c_{r(a)}\right\}$ with $\eta_{i}=\left.\eta\right|_{\left(\widetilde{X}, c_{i}\right)}:\left(\tilde{X}, c_{i}\right) \rightarrow$ $\left(X_{i}^{\prime}, h(a)\right)$ biholomorphic, we can define $\tilde{h}:\left(\tilde{X}, b_{i}\right) \rightarrow\left(\tilde{X}, c_{i}\right)$ by the formula $\tilde{h}=\eta_{i}^{-1} \circ h \circ \bar{\eta}_{i}$. Therefore, we have extended $\tilde{h}^{\prime}$ to a homeomorphism $\tilde{h}: \tilde{X} \backslash \tilde{X}_{s} \rightarrow \tilde{X} \backslash \tilde{X}_{s}$ where $\tilde{X}_{s}$ is a discrete subset of $\tilde{X}$. Thus we need to extend $\tilde{h}$ across $\widetilde{X}_{s}$. If $b \in \widetilde{X}_{s}$, then $\eta\left(\tilde{h}\left(\widetilde{X}_{\text {reg }}, b\right)\right)$ is a connected component of $(X \backslash S(X), h(\eta(b)))$ so the closure is an irreducible component of the germ $(X, h(\eta(b)))$. Thus the points of $\eta^{-1}(h(\eta(b)))$ are in one-to-one correspondence with the irreducible components of $(X, h(\eta(b)))$. Let $\tilde{h}(b)$ be the point corresponding to $\overline{\eta\left(\tilde{h}\left(\tilde{X}_{\text {reg }}, b\right)\right)}$. This completes the construction of $\tilde{h}$ and from the method of construction it is clear that $\tilde{h}: \tilde{X} \rightarrow \tilde{X}$ is a homeomorphism, and the theorem is proved.

\section{REFERENCES}

1. W. A. Adkins and J. W. Leahy, A topological criterion for local optimality of weakly normal complex spaces, Math. Ann. 243 (1979), 115-123.

2. D. Mumford, The topology of normal singularities of an algebraic surface, Publ. Inst. Hautes Et. Sci. No. 9, Paris (1960).

3. J. H. M. Steenbrink and J. Stevens, Topological invariance of the weight filtration, Nederl. Akad. Wetensch. Indag. Math. 46 (1984), 63-76.

Department of Mathematics, Louisiana State University, Baton Rouge, Louisiana 70803 